2016-2017. Most of these sites now demonstrate earlier integration of supportive care within cancer through implementation of ESC - some have shown very significant increases in early referrals. In some sites, data points to an impact on reducing emergency admissions.

Conclusions NHS England (NHSE) continues to build the case that ESC delivers improved value - reduced healthcare costs, improved QoL and optimises treatment decision making. Data collection is ongoing nationally and will inform national cancer policy and NHSE strategy. Formal research evaluation of ESC is expected begin in early 2018.

\section{IMPLEMENTATION OF SERIOUS ILLNESS CARE PROGRAMME UK FOR PATIENTS WITH ADVANCED CANCER: FEASIBILITY STUDY}

Tamsin McGlinchey, Stephen Mason, Alison Coackley, Anita Roberts, Maria Maguire, Justin Sanders, Francine Maloney, Susan Block, John Ellershaw, Peter Kirkbride. Marie Curie Palliative Care Institute in Liverpool, Clatterbridge Cancer Centre NHS Foundation Trust, Ariadne Labs Brigham and Women's Hospital and Harvard T.H. Chan School of Public Health

\subsection{6/bmjspcare-2018-ASPabstracts. 187}

The Serious Illness Care Programme is a complex intervention developed in the US to improve communication between clinicians and patients with serious illness. In collaboration with Aridane Labs the programme has been developed for use within the UK, with pilot implementation funded by NHS England. Within the programme, clinicians are trained in use of a Serious Illness Conversation Guide (the guide), to structure conversations with patients/families. A feasibility of the UK programme is underway, within one North West cancer centre.

Aim To present interim results from the feasibility study

Methods Data on the use and experience of the UK programme, from the perspective of clinicians and patients

Quantitative Data: Questionnaires:

Clinicians - pre/post training:

- confidence;

- attitudes to care of the dying;

- acceptability of serious illness care programme

Patients - pre/post serious illness conversation:

- Quality of clinical communication;

- Quality of life;

- Anxiety;

- depression.

Qualitative Data: In depth interviews:

Clinicians: experience of the UK programme, education and training and engaging patients in serious illness conversations

Patients: experience of engaging in serious illness conversations, including their acceptability of the approach

Results Quantitative Data: Results will be presented to assess the feasibility and acceptability of the UK programme:

- Proportion of patients identified conversations and participation in study measures

- Illustrate any indicative improvements in communication and patient outcomes.

Qualitative Data: Thematic Analysis to explore perceptions and experience of the programme: results will highlight main themes, such as: Conversation Value; Holistic and Compassionate; Opening up conversations with family members; Breaking the ice; Timing of conversation and Prognosis.

Conclusion Results from this study suggest these conversations promote shared decision making and individualised care planning, and espouse 'holistic' and 'person centred' care. Further research and evaluation is required to understand the effect of the UK programme on patient outcomes.

\section{E ASSESSING THE IMPACT OF ENHANCED SUPPORTIVE CARE USING THE INTEGRATED PALLIATIVE CARE OUTCOME SCALE (IPOS) AT THE CHRISTIE NHS FOUNDATION TRUST}

Lisa La Mola, Mark Warren, Richard Berman, Shameem Lilley, Phil Higham, Hannah Clare. The Christie NHS Foundation Trust

10.1136/bmjspcare-2018-ASPabstracts. 188

Enhanced Supportive Care (ESC) is a new UK initiative that integrates supportive care within oncology. At its heart is better access to expertise in managing the adverse effects of cancer and cancer treatments to enhance the patient experience and reduce the need for hospital admission.

ESC is award winning and has been recognised nationally by NHS England.

The aim of this audit was to measure the impact of ESC using the Integrated Palliative Care Outcome Scale (IPOS). This is a validated tool that can be used in clinical audit, research and training to measure symptom burden.

Method 47 patients referred into the supportive care service, were randomly selected to complete the IPOS tool within 3 areas (inpatients, outpatients and trials unit) during May to October 2016. For all areas a baseline IPOS was completed prior to the initial assessment by supportive care. Subsequent IPOS were completed approximately 3 days later for inpatients. Outpatients and trial patients were reviewed at various time points depending on the timing of their follow up appointments. Outpatients had an average follow up of 41 days. Trial patients had an average follow up of 15 days.

Results The overall IPOS score is the sum of the score of each of the 17 questions and can range from 0-68. A reduction in the IPOS score demonstrates an improvement in physical and psychological patient symptom burden. 16 inpatients completed the IPOS and demonstrated a 50\% improvement in overall mean symptom score. 5 experimental cancer medicine patients completed the IPOS and demonstrated a $49 \%$ improvement in overall mean score. 26 oncology outpatients completed the IPOS and demonstrated a $2.5 \%$ deterioration in overall mean score, demonstrating a possible correlation between frequency of supportive care review and symptom burden.

\section{UK AND IRISH HOSPICE AND SPECIALIST PALLIATIVE CARE UNIT EXPERIENCE OF ASSISTED VENTILATION IN MOTOR NEURONE DISEASE}

Aoife Gleeson, Faye Johnson. Aneurin Bevan University Health Board

10.1136/bmjspcare-2018-ASPabstracts.189 
An increasing number of patients with motor neurone disease (MND) in the UK and Ireland are using assisted ventilation to control symptoms and prolong life. A small proportion of these are initiated on long term tracheostomy ventilation (TV). NICE guidelines dictate that MND patients should routinely receive specialist palliative care input, yet research into the management of TV MND patients in the hospice setting is limited. Objective

The aim of this study was to explore the extent to which hospices and specialist palliative care units (SPCUs) in the UK and Ireland currently manage MND patients using assisted ventilation, especially TV.

Methods A literature review was used to guide the development of an electronic questionnaire which was sent to medical directors or lead physicians in all hospices/specialist palliative care units in the United Kingdom and Republic of Ireland. the questionnaire was sent to 184 units.

Results The response rate was 41.3\% $(n=76)$. 97\% units admit MND patients on non-invasive ventilation (NIV) but only 33\% admit TV MND patients $(n=22)$. However $40 \%$ of respondents indicated that community palliative care support was provided to TV MND patients. 61 respondents indicated adequate expertise in the management of NIV, while only 6 indicated adequate expertise in managing TV. While the majority of respondents (96\%) indicated that Advance care planning was routinely offered to the MND patients, less than half routinely included conversations regarding ITU admission (40\%) or invasive ventilation (39\%). Only $11(14 \%)$ of respondents indicated having personal experience of withdrawal of TV in MND patients.

Conclusion This study has highlighted a perceived lack of expertise in the management TV MND patients within hospices/SPCUs with less than half of units allowing admission for TV MND patients. There is a need for further research in this area.

\section{EVALUATION OF CAN-GUIDE (CANCER-GOAL USE IN DECISIONS): A DECISION MAKING SUPPORT PACKAGE TO ENHANCE SHARED DECISION MAKING IN PROGRESSING CANCER}

Grant Punnett, Leanne Howieson, Charlotte J Heaven, Sally Taylor, Carole Mula, Wendy Makin, Janelle Yorke. The Christie NHS Foundation Trust, University of Manchester

\subsection{6/bmjspcare-2018-ASPabstracts. 190}

Introduction National guidelines recommend that shared decision making be employed where several treatment or supportive care options are available to the patients with cancer. Shared decision making is associated with positive outcomes for patients including reduced decision regret surrounding their treatment and reduction of unrealistic expectations. Optimising shared decision making in situations where cancer is no longer curable may facilitate the discussion about not only the aims of treatment but also the patient's life goals and how their treatment options may affect these.

Aim to evaluate the Cancer-Goal Use In DEcisions (CanGUIDE) web-based communication/decision aid as a resource for facilitating goals of care conversations and shared decision making in the context of incurable cancer.

Method A pre-test post-test pilot study to assess the benefit of Can-GUIDE on patient experience and shared decision making. The quality of the decision making process is currently being assessed by determining the effect on patient decision self-efficacy, decision conflict, preparation for decision making and perceived involvement in shared decision making with their clinician. Oncology clinician opinion will be sought to determine whether patient responses to the package helped guide GOC conversations within their consultations. Furthermore, GPs will be surveyed to determine the usefulness of receiving a tool documenting GOC conversations within oncology consultations. The views of all participant groups surrounding the usability and acceptability of Can-GUIDE are also being investigated.

Results Currently, 14 patients have been recruited for the study with 4 withdrawn due to progressing disease or noncompliance with protocol. Data collection is due to complete March 2018 with a total of 40 patients recruited.

Conclusions If our pilot results demonstrate the potential value of Can-GUIDE a fully powered randomised controlled trial will be conducted to tests its effectiveness on improving patient-centred outcomes.

\section{BEYOND EARLY INTERVENTION - INTEGRATED WORKING IN A BRAIN TUMOUR CLINIC} ${ }^{1,2}$ Carolyn Campbell, ${ }^{3}$ Josephine Peck, ${ }^{3}$ Victoria Teague, ${ }^{2}$ Fiona Minear, ${ }^{2}$ Glad Baldry,
1,2 Jane Gibbins, ${ }^{1}$ Deborah Stevens. ${ }^{1}$ Cornwall Hospice Care; ${ }^{2}$ Royal Cornwall Hospitals
Trust; ${ }^{3}$ University of Exeter Medical School

10.1136/bmjspcare-2018-ASPabstracts. 191

Background An increasing body of evidence supports early palliative care involvement in oncology as improving quality of life, advance care planning and decision making. Patients with brain tumours experience high symptom, social and psychological burden and those with lower grade tumours may struggle to access palliative care services earlier in their disease trajectory. This tumour site has not been studied in any previous work on early intervention or joint working. This service review looks at a joint oncology/palliative care, consultant delivered, brain tumour clinic.

Methods Records of 133 consecutive consultations (82 patients) were analysed for demographics, diagnosis, clinician seen, primary purpose of appointment and additional topics addressed. For a cohort of patients from each diagnostic group, all consultations since diagnosis were reviewed to establish any patterns of engagement over time and disease progression.

Results $43.6 \%$ of consultations were with the palliative care consultant (PCC). Longitudinally, $81.8 \%$ of patients saw the PCC at least once. Whilst PCC (60.3\%) and oncologist (62.6\%) had a similar proportion of complex consultations (those dealing with at least one additional topic) for the oncologist these were concentrated in new patient consultations and treatment decisions (63\%) and for patients with glioblastomas (60.1\%); whereas for the PCC, complex consultations were distributed across all tumour types except pituitary and predominantly occurred in routine reviews (76.5\%). Levels of complexity were comparable in patients with lower grade gliomas or meningioma to those with glioblastomas.

Conclusions This analysis demonstrates a high level of complex need in patients with brain tumours and particularly for those with lower grade tumours over the very prolonged course of their disease. Integrated working appears to facilitate early involvement of palliative care and collaborative care, 